\title{
Problem-solving in mathematics with the help of computers
}

\begin{abstract}
ANDRÁs KovÁCs
Abstract. One of the most important tasks of the didactics of mathematics is the describing of the process of problem-solving activity and problem-solving thinking. The psychological theories concerning the problem-solving thinking leave the special demand of school subjects out of consideration, and search for connections of universal validity. In this article we attempt to connect an abstract theory of psychology concerning problem-solving thinking and a more practical conception of the problem-solving activity of mathematics, which is based on Polya's idea. In this way we can get a structure of problem-solving, which has scientific bases and at the same time it is useful in computer aided learning. Our result was developed and tested in Hungary so this is suitable especially for the Hungarian conditions of mathematics teaching.
\end{abstract}

Key words and phrases: problem-solving, thinking phases, thinking operations, computer aided learning.

ZDM Subject Classification: C39.

\section{Agreements}

In this article we will often use the concepts of problem and task. These two words of psychology have different meanings. (Task is a more general concept than problem.) Since we use these words in connection with mathematics teaching, and often in this environment task and problem have the same meaning, we shall consider these two words the same. The common characteristic of these words in our approach is the following. Task and problem have got such a goal that we do not know how to reach. 
Because of the previous agreement, we use the word thinking for problemsolving thinking. It deduces new connections from the well-known data of the problem. The reason of this procedure is that when a teacher applies computers in a mathematics lesson, then this teacher mainly uses it for intellectual activity.

We refer to mathematical problems in our example. But our statements are valid for all those subjects, in which the main type of activity is the intellectual activity.

\section{Raising the problem}

The initiation of computer aided learning would result

(1) the confirming of the motivation;

(2) the connecting the divergent-type of problem-solving into the educational process;

(3) the increase of the achievement and activity of students.

But these conditions will become reality, if not the instinctive but the conscious aspect directs the realization of the use of computers. It would be very important that the computer aided learning should have its own didactics in order to help those teachers who apply computers in their work.

\section{The structures of problem-solving}

\subsection{The phases of problem-solving thinking}

Until the middle of the 20th century, the situation of the didactics of mathematics was the same as the situation of the didactics of computer aided learning at present. The didactics of computer aided learning has got a lot of excellent results, a few interesting teaching methods, but it has not got a general, unified theory.

George Polya was the first who called attention to the importance of psychology in the didactics of mathematics. (Perhaps his step was the accelerant which started the abrupt development of the didactics of mathematics.) He summarized those experiences, which he had collected formerly in connection with the structure of problem-solving in mathematics.

Polya suggested such a description of the process of problem-solving in mathematics which consists of four steps. These are the following: 
P1. understanding the problem;

P2. devising a plan;

P3. carrying out the plan;

P4. looking back.

Because this system consists of only a few steps, he divided on the process of problem-solving with a lot of questions and instructions. So this structure became very useful for every student and mathematics teacher. As he built his system not with scientific method, it may be further completed, if new mathematical problems should arise. Schoenfeld performed an important transformation with Polya's system.

The application of the computers would modify the original idea considerably, and would raise a lot of problems. Therefore it would be worth initating a new system. For the sake of scientific requirements we had to look for a psychological theory which is supported by experiments. We examined several theories concerning problem-solving and cognitive thinking. Since the middle of the eighties the efficiency of the education of mathematics has been decreasing in Hungary, so we have to find such an idea, which is accepted by the most Hungarian mathematics teachers.

In 1999 we asked 42 teachers, who had certain contacts with the University of Debrecen, about this question. All the 42 mathematics teachers voted for the theory which is the most similar to Polya's conception. In our opinion this theory which was elaborated by one of the most recognized Hungarian psychologists, Ferenc Lénárd, is suitable for supplying the most successful result under the special Hungarian conditions.

After Lénárd had analysed 3426 steps of 135 reports, he concluded that problem-solving thinking could be divided into the following phases:

L1. fact-finding;

L2. modification of the problem;

L3. suggestion for problem-solving;

L4. criticism;

L5. mentioning accessory elements;

L6. wonder, delight;

L7. annoyance;

L8. scepticism;

L9. giving up the activity. 
Let's examine these phases of thinking and compare these steps with Polya's four steps.

According to Lénárd, fact-finding is the determination of the data, connections of the given problem. So we should say, that the two steps, i.e. fact-finding and understanding the problem are the same.

If we change the data and the connections, then we get the modification of the problem. This would lead us to the solution of the problem. According to Polya, one of the most important ways of the devising a plan is looking for a related, a more general, a more special or an analogous problem, i.e. the modification of the problem. Since we use the method of the modification of the problem in the hope of solving it, we can consider these phases a part of devising a plan.

According to Lénárd's opinion suggestion for problem-solving means the process of devising a plan. Carrying out the plan is a phase of the activity which does not appear in Lénárd's system. Carrying out the plan is not a part of the problem-solving thinking, but it is a part of the problem-solving activity. (Schoenfeld modified Polya's system also in this way. He left out carrying out the plan from the phases of thinking.)

Criticism contains all the observations, which are related to fact-finding and suggestion for problem-solving. Polya's phase named looking back means exactly the same.

Mentioning accessory elements is observable at such remarks of the suggestion for problem-solving which are not connected with the problem in hand. In our opinion this step shows the mistake of the suggestion for problem-solving. It would be interesting for a psychologist, if somebody wrote down inappropriate things during the process of problem-solving. But it is information of no use for an expert of the didactics of mathematics.

Wonder, delight, annoyance, scepticism and giving up the activity, i.e. the affective categories can hardly be shown in the school-work of students. On the other hand, a part of psychologists query, whether they are independent phases of problem-solving thinking, or not. When we analysed in an experiment earlier the mistakes of mathematical thinking, we experienced that emotions appear in the thinking, but these operations can only help or prevent the developing of thinking phases. We agree with Polya's views that emotions do not belong to the phases of problem-solving.

Summarizing our results till now we have obtained although psychology has got a richer structure of problem-solving than mathematics didactics, its elements 
of teaching - fact-finding, suggestion for problem-solving and criticism - correspond to the phases found in Polya's idea. This fact shows that Polya's system is applicable not only for mathematics but for all the school-subjects which show similar student's activity as mathematics. (We will consider the same L1 as P1, L3 as P2 and L4 as P4 further on because of the previously written reasons. So we use for their marks only the numbers 1, 2 and 3. Since we will deal with Lénárd's system in the following chapters, so we will use Lénárd's names. For this reason the number 1 means L1, 2 means L3 and 3 means L4.)

\subsection{The operations of problem-solving thinking}

Lénárd was absorbed in the theory of problem-solving thinking for decades. According to his theory thinking is always the result of two processes taking place on a different level. The phases of thinking mean steps relating to the whole thinking process. The construction of the macrostructure include the phases of thinking. On the other hand, however, the steps of thinking are influenced not only by the whole thinking process, but by the small surroundings of the individual steps, the parts of the so-called microstructure as well. In this way Lénárd got to the following operations in thinking:
A) analysis;
B) synthesis;
C) abstracting;
D) comparing;
E) comparing of abstract data;
F) comprehension of relations;
G) addition;
H) generalizing;
I) putting things and relations concretely;
J) putting things and relations in order;
K) analogy.

The greatest trouble with the structure mentioned above is that there are a few thinking steps which are special cases of other thinking steps. Since the whole thinking process can be described with the leaving out the unnecessary thinking steps, we do not take these into consideration.

Let's take the situation of the microstructure in a more detailed way. 
Analysis is an operation of thinking which resolves a whole thing into components.

Abstracting emphasizes a property of a whole thing, which is not considerable as an independent unit. Since this operation can be observed when we resolve a whole thing into components, so - agreeing with Rubinstein in this question - we consider abstracting a special part of the analysis.

Synthesis and analysis are the opposite parts of the same thinking process. Synthesis unites the parts resolved by the analysis. As a result of this union we can get the connections among the parts of the whole thing.

Comprehension of relations is the definition of a relation which exists between two things or two notions. (Such relations are for example similar, opposite, less, greater, equal, etc.)

Comparing and comparing of abstract data are operations which show identity or difference of certain things or notions. These relations are parts of the relations mentioned above. So we can leave these operations.

Addition names a thing or notion with full knowledge of a relation and a thing or notion included in this relation. Lénárd considered generalizing and putting things and relations concretely as cases of addition, so we leave the last two operations out of consideration.

Putting things and relations in order is an operation which chooses the suitable ones from the group of data, notions, connections.

Analogy means that at first we have to find connections among the given notions, relations or data and other notions, relations or data which we think similar to one another. Then we apply the solution of the related case for the original problem.

If we consider only the 6 main thinking operations, - analysis, synthesis, comprehension of relations, addition, putting things and relations in order and analogy, which we will mark with lower cases in alphabetical order - then we can organize experiments in connections with the process of problem-solving thinking. We started an experiment in 1995. In this experiment we examined mistakes of problem-solving in mathematics learning. We reviewed 1274 bad solutions in algebraic exercise, and found 1509 mistakes in these exercises. We succeeded to place all the mistakes in our system. This fact shows that our method can describe the process of problem-solving thinking. 
The system of problem-solving thinking can be represented with a table.

\begin{tabular}{l|c|c|c|c|c|c}
\hline & $\begin{array}{c}\text { (a) } \\
\text { analysis }\end{array}$ & $\begin{array}{c}\text { (b) } \\
\text { synthesis }\end{array}$ & $\begin{array}{c}\text { (c) } \\
\text { comprehension } \\
\text { of relations }\end{array}$ & $\begin{array}{c}\text { (d) } \\
\text { addition }\end{array}$ & $\begin{array}{c}\text { (e) } \\
\text { putting things } \\
\text { and relations } \\
\text { in order }\end{array}$ & $\begin{array}{c}\text { (f) } \\
\text { analogy }\end{array}$ \\
\hline 1. fact-finding & & & & & & \\
\hline $\begin{array}{c}\text { 2. suggestion for } \\
\text { problem-solving }\end{array}$ & & & & & & \\
\hline 3. criticism & & & & & & \\
\hline
\end{tabular}

Figure 1. The structure of problem-solving

\section{The process of problem-solving}

If we want to continue our work in accordance with Polya's idea, we have to plan our questions and instructions concerning problem-solving thinking with the help of our table. Let's give a few questions and instructions for example. (The lower cases refer to the thinking operations of the Figure 1.)

\section{Fact-finding}

a) What is the problem? What are the data? Divide the data and the problem into parts.

b) Are the data sufficient for problem-solving?

c) Is it connection among the data? Or contradictory?

d) What kind of connection is it among the data?

e) Is it possible to group the data according to a kind of point of view?

f) Do you know related data to other data of a well-known problem?

\section{Suggestion for problem-solving}

a) Is it connection among the data and certain parts of the solution?

b) Is it possible to deduce from the data or the structure of the solution something?

c) Could you solve a part of the problem?

d) Is it possible to determine those tools, with which we can solve the problem starting from the data?

e) Is it possible to order the steps, which are necessary for problem-solving?

f) If you cannot solve the proposed problem try to solve first some related problem. Could you imagine a more accessible related problem? A more general problem? A more special problem? An analogous problem? 
3. Criticism

a) Did you use all the data?

b) Is the structure of the solution good?

c) Does the solution answer our previous expectations?

d) Can you derive the solution differently?

e) Could you choose a few important details of the solution?

s) Do the related (special, general) results of problems verify your solution?

\section{Informatical features of problem-solving thinking}

The connecting of the computer in the mathematics teaching does not change the structure of problem-solving, but it can increase the numbers of questions and instructions. In this way we can get, that the results of the (1)-(3) points of the $2^{\text {nd }}$ chapter will be realized.

We will mention a few examples below, which can complete our earlier system. (The numbers show the thinking phases and the lower cases refer to the thinking operations of the Figure 1.)

1.a) Have you got a suitable program for analysing data?

1.a) Could you use the computer in problem-solving?

1.b) Could you initiate new data in the process of problem-solving with the help of computers?

1.c) Do you know such a software with which can discover connections among data?

1.d) Could you verify connections among data with the help of computers?

1.e) Try to group the data with computers.

2.a) Try to get further data. Use computers.

2.b) Draw a figure or make a table with computers. Try to describe the connections among the well-known data.

2.c) Could you describe unknown connections among the data? Write these new connections in the figure or in the table.

2.d) Try to solve the problem with computers.

2.e) Order the characteristics of the problem. Write the characteristics in computer in such a way that these characteristics can be retrieve. 
2.f) Could you find a related problem in your booking?

3.a) Can you use computers for criticism?

3.b) Can you describe connections among the solution?

3.c) Could you find such a matter on the web which can connect with the solution of the problem?

3.d) Check the solution with computers.

3.e) Make a web-page to show the process of problem-solving.

3.f) Look for related results on the web. Can these results prove your solution?

\section{Examinations}

We have tried the following exercises out in teacher training courses which we organized from 1999. These teacher training courses consist of 360 hours and last two years. The participants of these teacher training courses learn modern results of pedagogy, psychology, mathematics and didactics of mathematics. The teachers, who attend these courses can try several activities which are connected with the using of computers through these exercises. Such an activity computerized drawing, counting, formal working or research working with the help of the web, creating hypothesis, critics, etc.

We can find the mathematical base of these examples in a book, which title is Calculus of Variations written by Lavrent'ev and Lyusternik. We can read here about the $J$-hyperbolas. If given two points $F_{1}$ and $F_{2}$ then a hyperbola usually is defined as the set of all points $P$ with $\left|P F_{1}-P F_{2}\right|=2 a$. In complete analogy now two lines $\Gamma_{1}$ and $\Gamma_{2}$ are given. Then a $J$-hyperbola is defined as the set of points $P$ with $\left|d\left(P \Gamma_{1}\right)-d\left(P \Gamma_{2}\right)\right|=2 a . d\left(P \Gamma_{i}\right)$ means the distance between $P$ and $\Gamma_{i}$. In this chapter different cases (in respect of signs) are discussed. Finally it turns out that eight rays occur (Figure 3). So a mistake, an incompleteness in [5] was discovered. At the end of this chapter a new problem is posed. What about the case that $\Gamma_{1}$ and $\Gamma_{2}$ both are circles or $\Gamma_{1}$ a circle and $\Gamma_{2}$ a line?

Now we will write the previous things in detail according to [5]. For knowing of the $J$-hyperbolas in the general case we have to give the curves $\Gamma_{1}$ and $\Gamma_{2}$ of the plane. Consider such extremal curves $\Gamma_{1}$ and $\Gamma_{2}$ from the $P$ points of the plane, which intersect $\Gamma_{1}$ and $\Gamma_{2}$ at right angles. Denote by $J\left(\Gamma_{1}\right)$ and $J\left(\Gamma_{2}\right)$ the lengths of the curves between the pairs $\left(P ; \Gamma_{1}\right)$ and $\left(P ; \Gamma_{2}\right)$. Then the set of points $P$ 
for those the difference $J\left(\Gamma_{1}\right)-J\left(\Gamma_{2}\right)$ is constant is called a $J$-hyperbola. (The authors did not use the signs of absolute values in connection with $J$-hyperbolas.)

In an example of this book the authors define a plane curve which is passing through a given point $\mathrm{A}$ of the plane. At this time let $\Gamma_{1}$ and $\Gamma_{2}$ be two lines. If we draw two lines perpendicular to $\Gamma_{1}$ and $\Gamma_{2}$ from different points $P$ of this curve then for the points of intersection $T_{P}^{\prime}$ and $T_{P}^{\prime \prime}$ - that is for the distances of the points and lines - the

$$
P T_{P}^{\prime}-P T_{P}^{\prime \prime}=A T_{A}^{\prime}-A T_{A}^{\prime \prime}=2 a
$$

equality must be true. (For the writing down of the previous equality we use that $J\left(\Gamma_{1}\right)=P T_{P}^{\prime}$ and $J\left(\Gamma_{2}\right)=P T_{P}^{\prime \prime}$.) The solution, according to the soviet authors, is a line passing through the $A$. We denote this line by $e$ on the Figure 2. We accept this solution as the $1^{\text {st }}$ hypothesis and then we tried to check the idea of the book with the teachers. (It is unusual that somebody set out from a wellknown solution as if it would be a fact-finding but this method was suitable for our purpose. We suggest the inductive method for problem-solving at schools. The softwares, which run according to Dynamical Geometry System, are used to this.) We did not use (1) for the starting of the examination. We considered the fact that in the school definition of hyperbolas there are signs of absolute values. Therefore we studied with the teachers that more general case, which includes the solution of the above-mentioned book. So our equality was

$$
\left|P T_{P}^{\prime}-P T_{P}^{\prime \prime}\right|=\left|A T_{A}^{\prime}-A T_{A}^{\prime \prime}\right|=2 a .
$$

We found that the solutions of (2) are two rays of the line $e$, which start from $G_{1}$ and $G_{2}$. So the curve which satisfies (1) can not be the whole line. We had to modify further the $1^{\text {st }}$ hypothesis. The solution is two rays of e according to the $2^{\text {nd }}$ hypothesis.

We took the $\Gamma_{1}^{\prime}$, which is parallel to $\Gamma_{1}$ for the examination. (It was the first step of suggestion for problem-solving.) The points of the $J$-hyperbola are at an equal distance from $\Gamma_{1}^{\prime}$ and $\Gamma_{2}$ because of the construction of $\Gamma_{1}^{\prime}$. In this way the points of the $J$-hyperbola lie on the bisector of $\Gamma_{1}^{\prime}$ and $\Gamma_{2}$. For the details we examined closely this problem. The points of that ray which starts from $G_{2}$ give the bisector of the angle $T_{P}^{\prime} P T_{P}^{\prime \prime}$, so because of $P T_{P^{\prime}}^{\prime}=P T_{P}^{\prime \prime}$, the next equality is true:

$$
P T_{P}^{\prime}-P T_{P}^{\prime \prime}=P T_{P}^{\prime}-P T_{P^{\prime}}^{\prime}=2 a
$$

Similarly, we can get the $P T_{P}^{\prime \prime}-P T_{P}^{\prime}=2 a$ equality, if the points $P$ are on the other ray of $e$. Consider those points $P$ of $e$, which can be found between the 


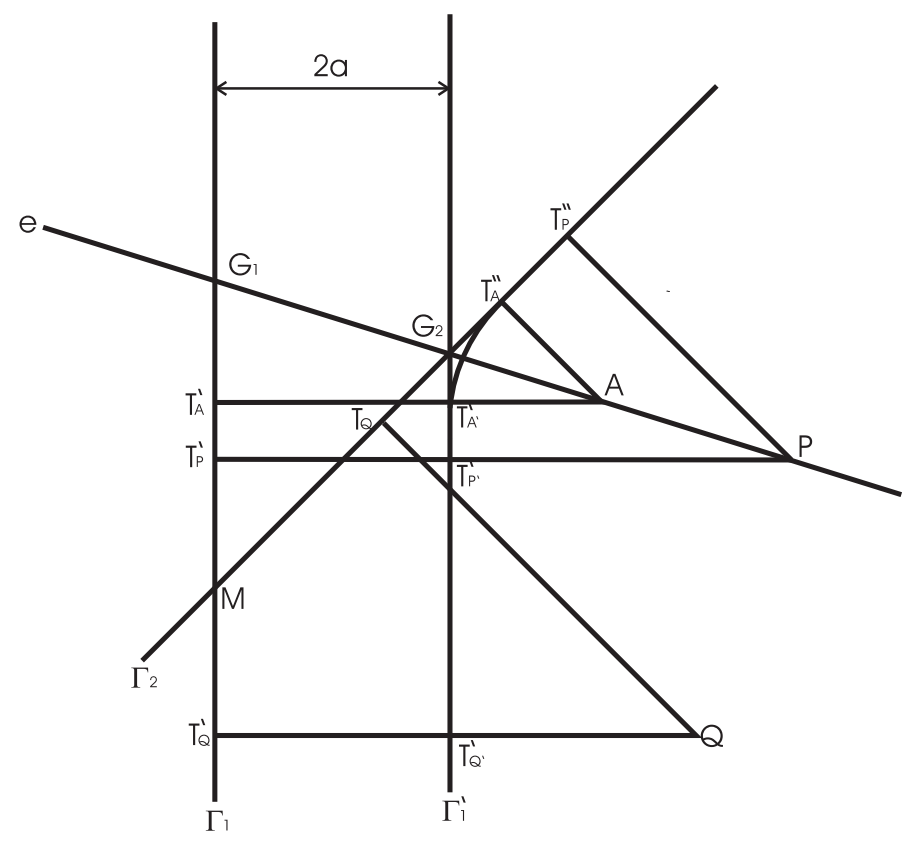

Figure 2. The J-hyperbola belonging to two lines

two rays. These points do not belong to our solution, because for these $P$, as we can see it easily, the next equality is true:

$$
P T_{P}^{\prime}+P T_{P}^{\prime \prime}=2 a .
$$

(We were inspired to initiate a new set of points by this new result later.)

It is easy to see that another point $Q$ of the plane does not belong to the set of points of the $J$-hyperbola. By using the $\left|Q T_{Q}^{\prime}-Q T_{Q}^{\prime \prime}\right|=\left|Q T_{Q^{\prime}}^{\prime}+2 a-Q T_{Q}^{\prime \prime}\right|=2 a$ equality we get that $Q T_{Q^{\prime}}^{\prime}=Q T_{Q}^{\prime \prime}$. So $Q$ is in the normal bisector of the $T_{Q}^{\prime \prime} T_{Q^{\prime}}^{\prime}$, which is parallel to the normal bisector of $T_{P}^{\prime \prime} T_{P^{\prime}}^{\prime}$. Because of this fact, the point $Q$ is not an element of the set of the $J$-hyperbola.

In that case when $\Gamma_{1}$ is parallel to $\Gamma_{2}$, the solution is a line, passing through the $A$ and parallel to the others. (If $\Gamma_{1}$ is parallel to $\Gamma_{2}$ then the solution is a line which passing through $A$ and parallel to $\Gamma_{1}$ and $\Gamma_{2}$.)

We could see that the process of criticism showed some kind of mistake. Since our results, which was taken with geometrical consideration, disagree with the solution of the book, we applied for computers. Because we should have liked to try a new learning method, therefore we turn to the Maple. This software 
run according to Computer Algebraic System. Namely it was treated during the teacher training courses.

We chose a simple example for checking. The $\Gamma_{1}: x=0, \Gamma_{2}: y=x$ and $A(4 ; 2)$ simple data lead equations of rays. We know that for point $P(x ; y)$ the equalities $J\left(\Gamma_{1}\right)=|x|$ and $J\left(\Gamma_{2}\right)=\left|\frac{x-y}{\sqrt{2}}\right|$ hold. It is easy to calculate that the distance of $A$ and $\Gamma_{1}$ is 4 , the distance of $A$ and $\Gamma_{2}$ is $\sqrt{2}$. By using these equalities and data we get that

$$
|| \frac{x-y}{\sqrt{2}}|-| x||=4-\sqrt{2}
$$

The participants of the experiment first made the decomposition of the absolut value traditionally and individually and then they co-operated in checking with Maple. We thought to get 8 sets of points because of the number of the signs of absolute values. And really we have got the next equations.

I. $x=-\sqrt{2} y+6+2 \sqrt{2}-y, \quad$ if $\quad y \leq \frac{2(3+\sqrt{2})}{\sqrt{2}+2}$,

II. $\quad x=-\sqrt{2} y-6-2 \sqrt{2}-y, \quad$ if $\quad y \leq-\frac{2(3+\sqrt{2})}{\sqrt{2}+1}$,

III. $x=\sqrt{2} y+10-6 \sqrt{2}-y, \quad$ if $\quad \frac{2(3 \sqrt{2}-5)}{-2+\sqrt{2}}<y$,

IV. $x=\sqrt{2} y-10+6 \sqrt{2}-y, \quad$ if $\quad-\frac{2(3 \sqrt{2}-5)}{\sqrt{2}-1} \leq y$,

V. $x=\sqrt{2} y+10-6 \sqrt{2}-y, \quad$ if $\quad y<\frac{2(3 \sqrt{2}-5)}{\sqrt{2}-1}$,

VI. $\quad x=-\sqrt{2} y+6+2 \sqrt{2}-y, \quad$ if $\quad \frac{2(3+\sqrt{2})}{\sqrt{2}+1}<y$,

VII. $x=\sqrt{2} y-10+6 \sqrt{2}-y, \quad$ if $\quad y \leq-\frac{2(3 \sqrt{2}-5)}{-2+\sqrt{2}}$,

VIII. $x=-\sqrt{2} y-6-2 \sqrt{2}-y, \quad$ if $\quad-\frac{2(3+\sqrt{2})}{\sqrt{2}+2}<y$. 
So we reached the $3^{\text {rd }}$ hypothesis which means eight rays. We can see the graphs of these eight rays in the next picture, which was made with Maple too.

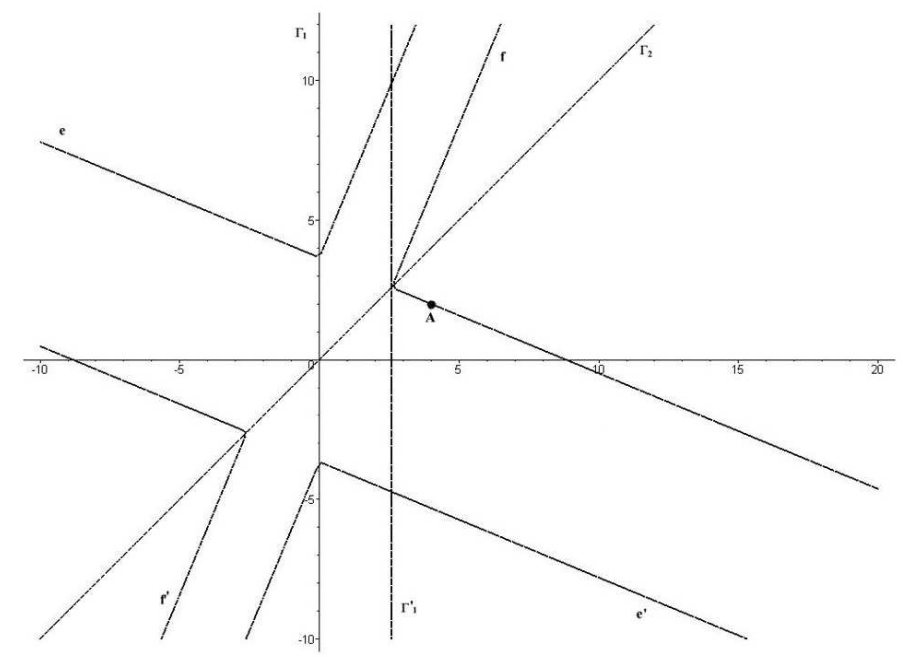

Figure 3. The drawing of J-hyperbola according to the computer

Now we see that every ray is parallel to the bisectors of $\Gamma_{1}$ and $\Gamma_{2}$, so the (2) equality is true for all of them. But among of these eight rays, there exists only two which lie on the line e passing through the $A$. ( $e^{\prime}$, which is parallel to $e$, is an image of $e$ reflected about the point of intersection of $\Gamma_{1}$ and $\Gamma_{2}$, so (2) holds for $e^{\prime}$ too because of the distance-preserving. But neither of the two rays of $e^{\prime}$ contain point $A$. The $f$ set of points, which consists of two rays, is an element of another bisector of $\Gamma_{1}^{\prime}$ and $\Gamma_{2}$. Since all points of this bisector are equidistant from the arms of the angle, so (2) holds for $f$ too. $f^{\prime}$ - similar to $e^{\prime}$ - is the image of $f$ reflected about point $O$. Neither $f$ nor $f^{\prime}$ contains point $A$.)

The detailed examination of the $3^{\text {rd }}$ hypothesis showed that the role of the bisectors are how important in this example. Thus we understood that the $3^{\text {rd }}$ hypothesis was not a wrong way. It could help us to differentiate our knowledge further. The $2^{\text {nd }}$ hypothesis gives the result after all.

We can examine that set of points which we call $J$-ellipse, in a similar way. The points of $J$-ellipse satisfy the

$$
P T_{P}^{\prime}+P T_{P}^{\prime \prime}=A T_{A}^{\prime}+A T_{A}^{\prime \prime}=2 a
$$


equality. The figure of this set of points is the segment $G_{1} G_{2}$, as we see below. It is easy to see that this segment lies on the bisector of $\Gamma_{1}^{\prime}$ and $\Gamma_{2}$. (We made mention of this fact in connection with Figure 2.) The detailed analysis and working out of this exercise was a home-work for teachers. We have checked it at the end of the teacher training courses.

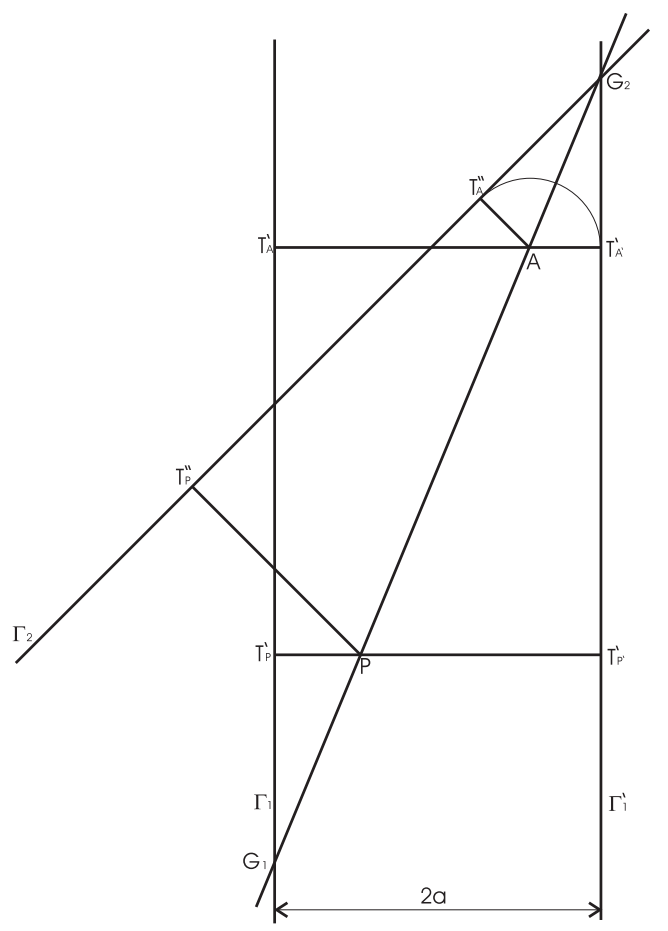

Figure 4. The J-ellipse belonging to two lines

The $J$-ellipse is on the bisector of the $T_{A}^{\prime \prime} G_{2} T_{A^{\prime}}^{\prime}$, since $T_{A^{\prime}}^{\prime} A G_{2}$ and $T_{A}^{\prime \prime} A G_{2}$ are congruent triangles. So if $P$ is a point of the segment, then the next equality is true:

$$
P T_{P}^{\prime}+P T_{P}^{\prime \prime}=P T_{P}^{\prime}+P T_{P^{\prime}}^{\prime}=2 a .
$$

If $\Gamma_{1}$ is parallel to $\Gamma_{2}$, then the solution - instead of the segment $G_{1} G_{2}$ - is a line which passing through $A$ and parallel to $\Gamma_{1}$ and $\Gamma_{2}$. 
With the help of the previously given $\Gamma_{1}, \Gamma_{2}$ and $A$, we got the next equation:

$$
\left|\frac{x-y}{\sqrt{2}}\right|+|x|=4+\sqrt{2} .
$$

The computer draws on the basis of this equation that figure which can see on Figure 5. The drawing of the computer little bit differs what we have got from our analysis. (We can see on this figure bisectors of $\Gamma_{1}^{\prime}$ and $\Gamma_{2}$, which are between $\Gamma_{1}$ and $\Gamma_{1}^{\prime}$ and the images of theirs reflected about the point of intersection of $\Gamma_{1}$ and $\Gamma_{2}$. But among of these segments, only the $e$ passing through the A.) This example shows the fact that the use of computers is not the aim, but only an instrument of the teaching.

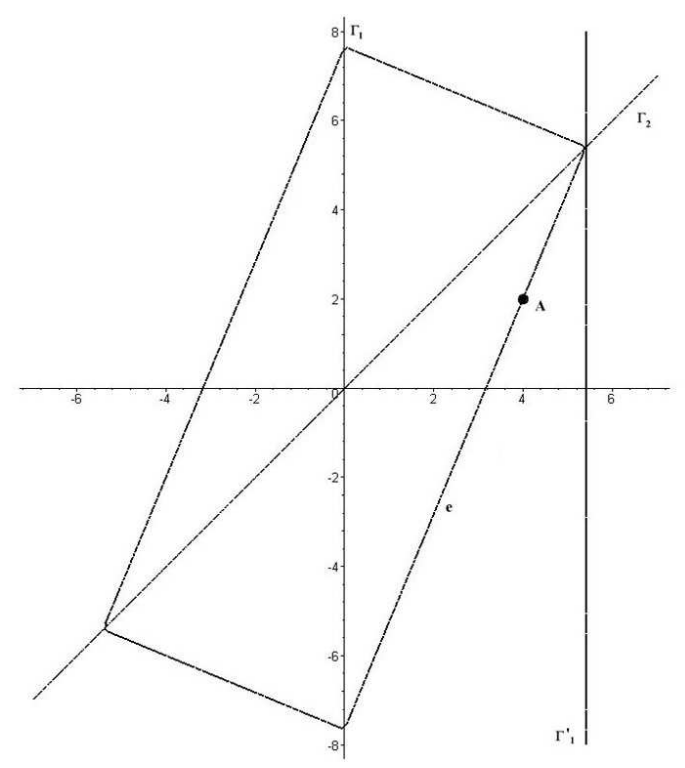

Figure 5. The drawing of J-ellipse according to the compute

Then we discussed with the teachers those cases, when both curves are circles or $\Gamma_{1}$ is a circle and $\Gamma_{2}$ is a line. So we got hyperbolas, ellipses and parabolas. 


\section{Experiences}

The exercises which are similar the previous ones give such problems, in which can occur every components of problem-solving thinking in connection with using of computers. Knowing the structure of problem-solving thinking, which we discussed earlier, we try to analyse these problems and we examined how can make studiable the problem-solving thinking for the students. We tried to plan our work by means of answering questions which were connected with problem-solving process in the experiment. We did it in that way how Polya advised in his books. But we had to transform the well-known questions because these questions must be suitable for computerize.

We drew our's conclusion from the experiment that it is very important to plan the whole process of problem-solving. Since we have to lead the students to this direction, we decided to write to our textbook such a guidance of problemsolving. We will do it through concrete example as we see it in Polya's books. It's important to show not only algorithms for solving certain exercises but we give instructions of universal validity.

We experienced that we must not believe in computers totally. On the other hand computers could make our work easier. With the help of computers we could go round and make clear the given problem.

The easy way of representation, the quick resolving an equation and the series of new-type checking made the experiment exciting and dynamical. So we could reach confirming of the motivation which was our aim (1).

In the obtaining of aim (2) the connecting the divergent-type of problemsolving into the educational process would be a very useful help the CABRI geometry software which makes speaking out conjectures and their generalization possible. But we did not use it in our experiment because we wanted to start problem-solving with a given result. The participants of the experiment remembered those experiences well that

- we must not believe in technical books even if these are the best;

- we must not believe in the best computer softwares totally.

We need checking always, Polya was quite right. We have to keep in view this thing when we teach students at school. We think this recognition would be considered the most important pedagogical aim of our experiment.

The aim (3), namely the increasing of the achievement and activity was fulfilled. Those participants who could not finish their idea alone or who considered reaching the aim too tired could be ready quickly with the help of computers. 
Finally we make mention of didactical aims of this experiment. We are convinced of the fact that our structure of problem-solving should be suitable for planning of the computer aided mathematics teaching. We use the phases of fact-finding, suggestion for problem-solving and criticism for the solving of the given problem as we mentioned earlier. We tried to check appearance of the 6 thinking operations through questions. (The teachers and me asked questions and answered these ones knowing our structure of problem-solving.)

We know that our method may not verify in that way as a mathematical theorem. But our idea proved to be true under the circumstances of the abovementioned example. The teachers use it very kindly. They recognized that with using this system their work become conscious.

Drawing a lesson from our experiment we summarize that in the teching of mathematics may use computers and it is our duty. For reaching the better result we have to apply to methods of didactics of mathematics and psychology. In our opinion knowing problem-solving thinking which is based on Polya's and Lénárd's idea can make more successful the computer aided mathematics teaching in Hungary.

\section{References}

[1] A. Ambrus, An Introduction to the Didactics of Mathematics, ELTE Eötvös Kiadó, Budapest, 1995 (in Hungarian).

[2] A. Ambrus and I. Hortobagyi, Problem-solving in teaching mathematics in Hungary, Der Mathematikunterricht 47(6) (2001), 6-17 (in German).

[3] K. J. Fuchs and E. Vásárhelyi, Problem - Analysis - Encoding - Testing, About program and data structures, Conference: International Conference on Technology in Mathematics Teaching, Klagenfurt, 2001, 189-194.

[4] Gy. Horváth, The matterful thinking, Tankönyvkiadó, Budapest, 1984 (in Hungarian).

[5] M. A. Lavrent'ev und L. A. Lyusternik, Lehrgang der Variationsrechnung, MoskauLeningrad: Staatsverlag für technisch-theoretische Literatur, 1950 (in Russian).

[6] F. Lénárd, The problem-solving thinking, Akadémiai Kiadó, Budapest, 1987 (in Hungarian).

[7] K. Mosonyi, Thinking Mistakes on Mathematics Lessons of Elementary School, Tankönyvkiadó, Budapest, 1972 (in Hungarian).

[8] I. Perjésiné Hámori, Application of CAS for teaching of integral-transforming theorems, Zentralblatt für Didaktik der Mathematik 35(2) (2003), 43-47.

[9] G. Polya, How to Solve It, 2nd edn., Princeton University Press, 1957. 
[10] G. Polya, Mathematics and plausible reasoning, Vol. 1, Induction and analogy in mathematics, Princeton, NJ, 1954.

[11] S. L. Rubinstein, Psychological-Thinking Examinations, Gondolat, Budapest, 1960 (in Hungarian).

[12] A. Schoenfeld, Mathematical Problem-solving, Academic Press, Inc., New York, 1985.

[13] R. J. Sternberg and T. Ben-Zeev, The Nature of Mathematical Thinking, Lawrence Erlbaum Associates, Publishers, Mahwah, NJ, 1996.

[14] E. Vásárhelyi, Mathematical representations of psychological situations, Conference: Gesellschaft für Didaktik der Mathematik, Klagenfurt, 2002, 499-502 (in German).

ANDRÁS KOVÁCS

INSITUTE OF MATHEMATICS

UNIVERSITY OF DEBRECEN

H-4010 DEBRECEN, P.O. BOX 12

HUNGARY

E-mail: kovacsa@math.klte.hu

(Received July, 2003) 